\title{
Information Design for Personas in Four Professional Domains of User Experience Design, Healthcare, Market Research, and Social Media Strategy
}

\author{
Kathleen Wenyun Guan \\ Division of Psychology and \\ Language Sciences \\ University College London \\ kathleen.guan.20@ucl.ac.uk
}

Soon-gyo Jung

Qatar Computing Research Institute

Hamad Bin Khalifa University

sjung@hbku.edu.qa

\author{
Joni Salminen \\ Qatar Computing Research Institute \\ Hamad Bin Khalifa University \\ jsalminen@hbku.edu.qa
}

\author{
Lene Nielsen \\ Business IT \\ IT University \\ lene@itu.dk
}

\author{
Bernard J. Jansen \\ Qatar Computing Research Institute \\ Hamad Bin Khalifa University \\ bjansen@hbku.edu.qa
}

\begin{abstract}
Practitioners in user-centric industries have increasingly recognized the applicability of personas. However, the methods used to create personas in different domains remain inconsistent and unsystematic. We analyzed 51 studies focused on designing personas for professional purposes and find the practice most prevalent in the user experience (UX) design, healthcare, market research, and social media strategy domains. Within these domains, UX design personas are characterized by their focus on user activity and goals, market research personas on customers' lifestyles, health personas on medical patients' physical symptoms, and social media strategy personas on interactions within online communities. We identify and compare the elements in the personas. Based on this, we provide guidelines for professionals interested in developing personas for recruiting users, understanding barriers to positive user experience, and building online communities, including how to represent persona details related to lifestyle, contexts of product usage, and scaling of online data.
\end{abstract}

\section{Introduction}

Personas, defined as "fictitious, specific, concrete representations of target users" [35:11], have increasingly become relevant for professionals interested in user-centric decision-making. User understanding is seen as critical for businesses [34] and data about users has become more accessible through online analytics platforms, social media profiles, and quantitative survey materials. This increase in data has led to more methods for persona creation, supplementing the qualitative methods such as ethnography and interviews [8], which were mainly used in the early days of the method. These new methods include quantitative approaches such as kmeans clustering [9] latent semantic analysis [30], matrix factorization [25], and mixed quantitativequalitative methods [32].

Nonetheless, there remains inconsistency among professional practices of persona content design, including guidelines on what standard information persona profiles should contain. The questions of what the persona creation protocol should be and what information should persona creators include have not been explored in-depth. Indeed, methodologies employed in the literature are often vaguely described and unsystematic, with little discussion on the justification behind the design choices [39]. Nielsen et al. [33] observed this shortcoming when analyzing and comparing the templates of user experience personas developed by Danish companies. Yet to our knowledge, no review has conducted to compare and contrast the applicability of personas for specific professional domains. To alleviate this gap, we propose the following research questions (RQs):

- RQ1: What are the professional domains that have most often used personas?

- RQ2: (a) What specific persona characteristics are included in the personas in each of these domains, and (b) how does this information compare with personas in the other domains?

- RQ3: What information should personas contain for different application purposes? 
We aim to identify the professional domains that most report to have used personas in the literature and discuss the (dis)similarities across domains. We highlight lessons learned to facilitate better persona application overall. We also provide practical design implications to recommend specific details that professionals should be aware of when developing personas, based on their needs and goals. Our objective is to enhance the current understanding of how professionals with a range of goals can benefit from personas.

\section{Methodology}

We identified the persona layouts analyzed in this paper using methods similar to a systematic review procedure [39], as we were unable to locate a comprehensive review of how personas have been applied to usage in professional domains. As such, it was necessary to systematically identify relevant case studies.

First, we conducted a general search for studies that created personas for research within professional contexts in two academic databases (Google Scholar and ACM DL). These literature searches were identical, consisting of references to persona creation ("personas," "user profile," "user type," "customer profile"). We also completed additional snowball sampling (examining the "references of references" [17:1065]) to account for papers discussing persona creation in the industry.

Our search yielded 146 unique articles that were screened in three separate title, abstract, and full-text review stages. Inclusion criteria at each stage were: full research articles, published in a peer-reviewed journal or conference, written in English, and details the development of personas using empirical and/or qualitative data. We further excluded studies that did not report from a specific professional context.

After this, 51 final papers remained, all of which were single case studies. We extracted the persona layouts for further analysis in a standardized data extraction form [49] to record the studies' title, authors, year of publication, professional domain category, professional domain subcategory (if relevant), and information in the persona profile. We captured the professional domain category and subcategories for each study. We determined these based on the (a) industry the study took place in, and the (b) job titles of those involved in the persona creation. We placed a particular emphasis on those that successfully applied the personas to enrich their work (as determined by their evaluation).

\section{Results}

We observed four professional domains that occurred most frequently: (a) user experience (UX) design (i.e., with regards to products and services), (b) market research (i.e., to identify or recruit new customers, (c) healthcare (i.e., the provision of care by medical professionals), and (d) social media strategy (i.e., strategies for the creation of digital content by "influencers" and other stakeholders to build online social communities). The frequency of studies in these domains is shown in Figure 1.

Most studies $(\mathrm{N}=24)$ applied personas to the domain of UX design. Market research and healthcare were the next top domains (both with $\mathrm{N}=9$ ). Studies applying personas to the domain of social media strategy were also prevalent $(\mathrm{N}=6)$.

Three studies had crossovers (and were thus double-counted) across the domains (a) UX design and health [46], (b) UX design and market research [48], and (c) market research and health [16]. We identified six studies pioneering the use of personas in unique contexts that were not prevalent enough to be considered under overarching professional domains, that explored applications of personas in film character development [7, 35], and psychology [10].

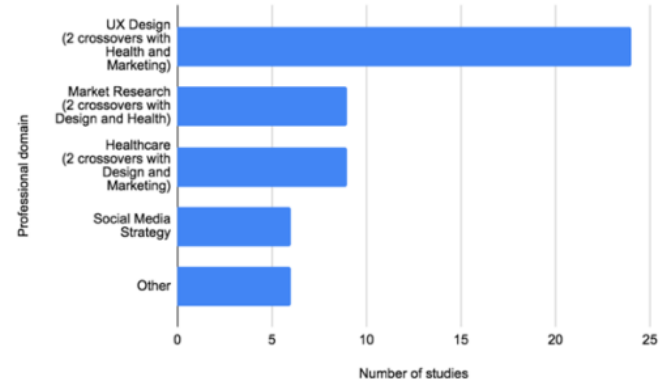

\section{Figure 1: Professional domains applying personas}

The next subsections will evaluate patterns in the persona profiles from the top four domains. First, representative examples and common information found in the personas within each domain are analyzed. The next section investigates the (dis)similarities of the informational content across the domains.

\subsection{User experience design personas}

There was great diversity among persona profiles created to inform UX design of products and services, such as studies focused on customers' preferences for software [4], e-commerce shopping websites [31], sports products [32], and video games [30]. All the 
persona profiles intentionally contained relevant information on current user activity and goals. Many of the UX design-focused studies also recorded information regarding demographics, personality, and lifestyle in the persona profiles.
As seen in Figure 2, the information found in these persona profiles is specific to current user goals and activity.

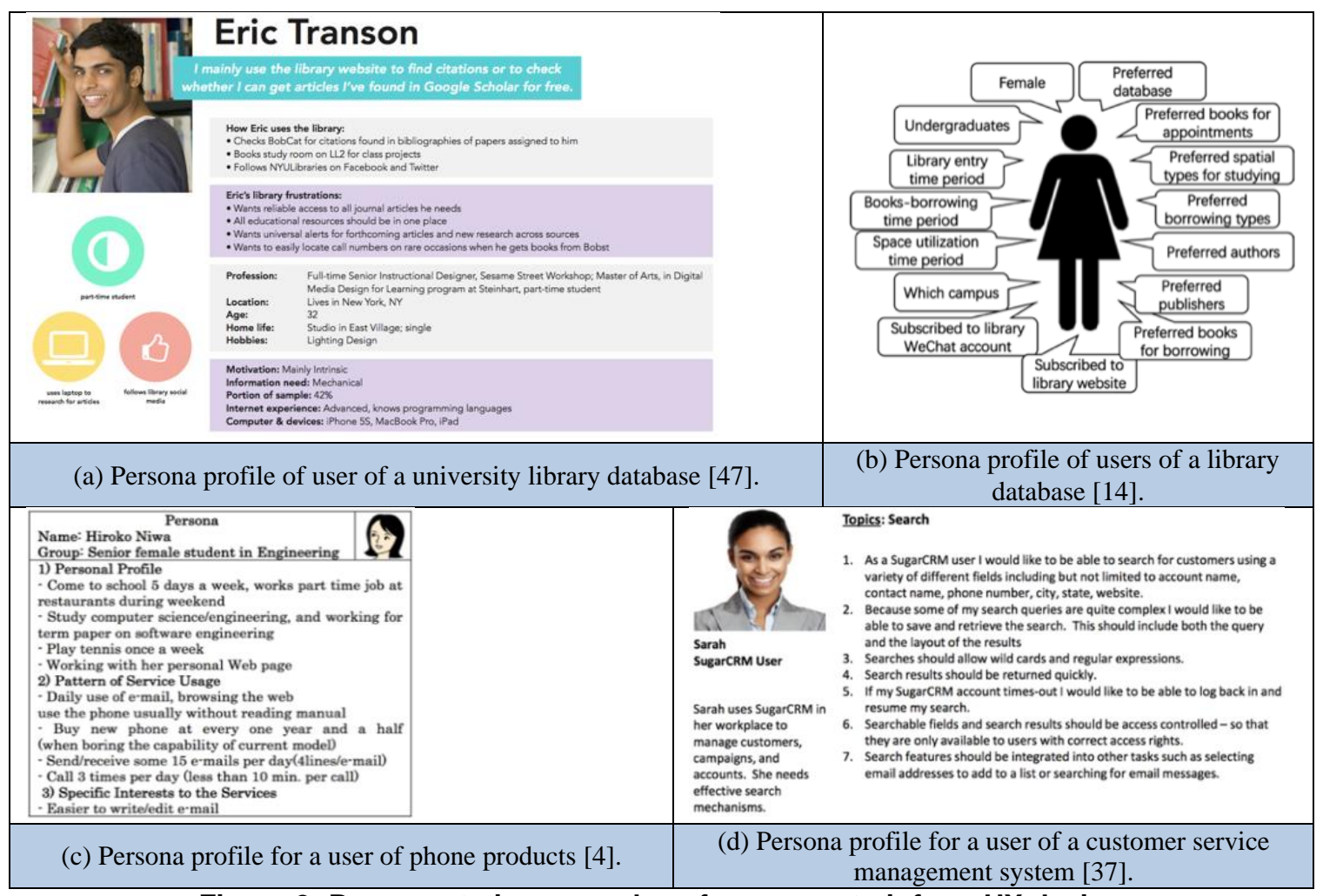

Figure 2: Representative examples of personas to inform UX design

For example, both personal profiles in Figures $2 \mathrm{a}$ and $2 \mathrm{~b}$ categorize specific details regarding the current use of and needs with regards to online library services, which directly align with the authors' interest in how to improve the university library portal for its users [14, 47]. Information regarding the persona's current library subscriptions, and devices used to access these subscriptions, are represented graphically to enrich UX designers' understanding of user activity.

The persona in Figure 2a details information related to the user's challenges and frustrations. Similarly, the persona profiles in Figures $2 \mathrm{c}$ and $2 \mathrm{~d}$ outline specific ways users interact with IT (mobile phone interface and customer service management system, respectively) and in the context of their job duties, as well as preferences for system's features and layout. The persona in Figure $2 c$ details the user's preferences for the mobile screen interface to be easier for writing emails.

\subsection{Healthcare personas}

Another professional domain where personas were applied was healthcare, specifically patient care. Among the persona profiles we identified in this domain, all were ultimately concerned with seeking to enhance medical professionals' understanding of their patients so they can ultimately provide more effective patient care. Authors of the studies discussed how the persona profiles could help medical professionals design tailored medical interventions to patient subpopulations represented by the personas [58]. To meet this aim, the healthcare patient personas contained relevant background information on patients' physical, mental, and cognitive health, in addition to psychological wellbeing, social activities, and current living environments.

As seen in Figure 3, this information could be represented in the form of a narrative and/or graphics. The persona profile in Figure 3 a contains narrative 
information on the relevant functional, psychological, technological, social, and behavioral information that could impact or imply potential medical conditions in the patient [19]. Figure 3b offers a similar — albeit simpler - graphical persona representation of a patient persona's fitness level, eating habits, weight, and psychographics [52]. Similarly, Figure 3c comprehensively details an elderly patient's household position, social life, income, and physical and cognitive traits [54]. One study additionally focused on tailoring healthcare advising/education appropriately to the needs of different coronary heart disease patient groups [51]. The authors posited that based on the personality and severity of symptoms, some patients might either benefit from or be adversely burdened when presented with information on the side effects of medical prescriptions. As such, this study uniquely included information on preferences regarding physician language style and the level of disease-specific information provided [51].

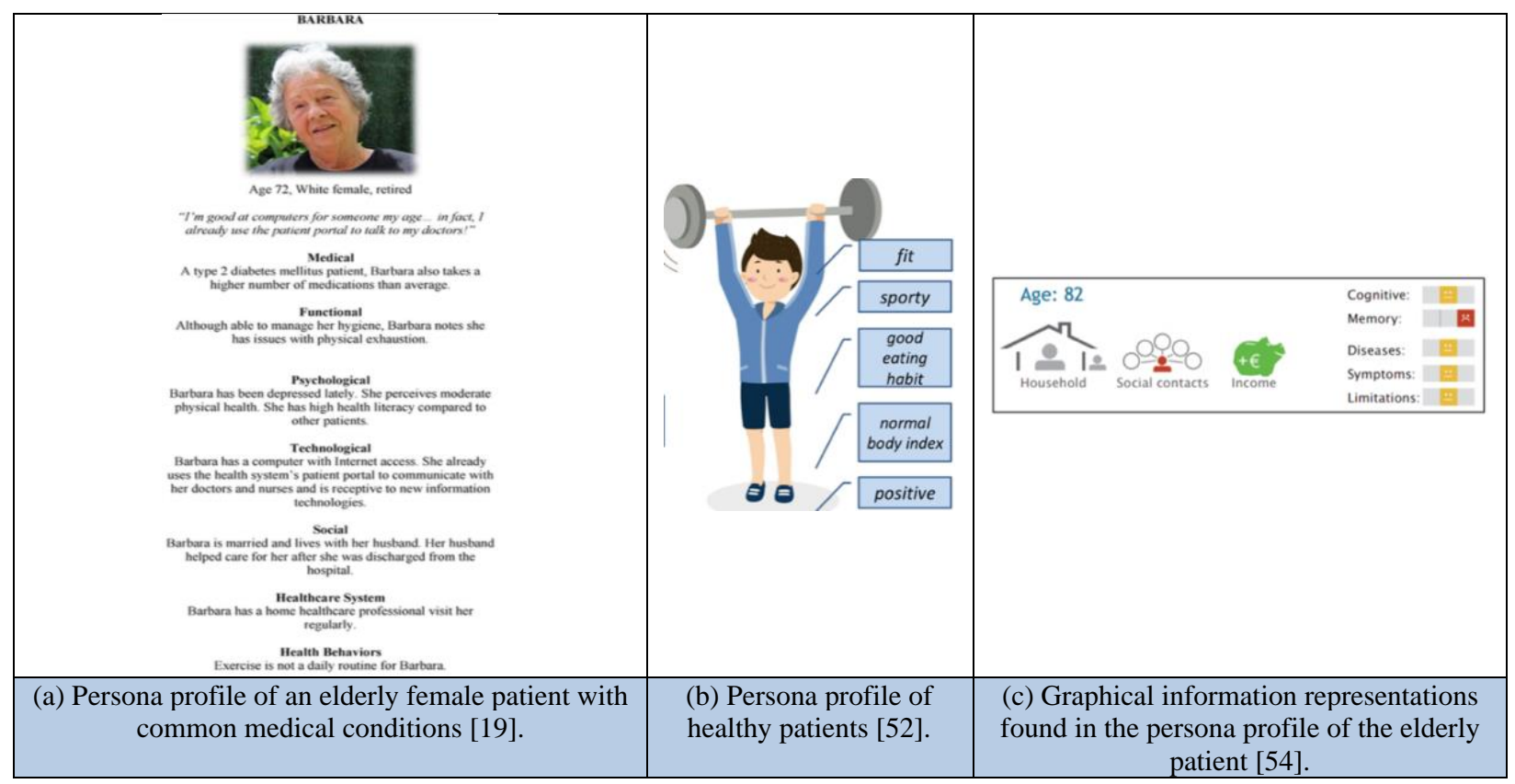

Figure 3: Representative examples of personas to inform healthcare

\begin{tabular}{|c|c|c|c|c|c|}
\hline $\begin{array}{l}\text { The revised persona : RPd (TabbleVII) } \\
\text { Taro (Male, } 29 \text { age) } \\
\text { Taro's favorite articles are about trivia } \\
\text { news. He does not use Netallica } \\
\text { frequently, but he shares interesting } \\
\text { articles on Facebook. }\end{array}$ & \multicolumn{2}{|c|}{ 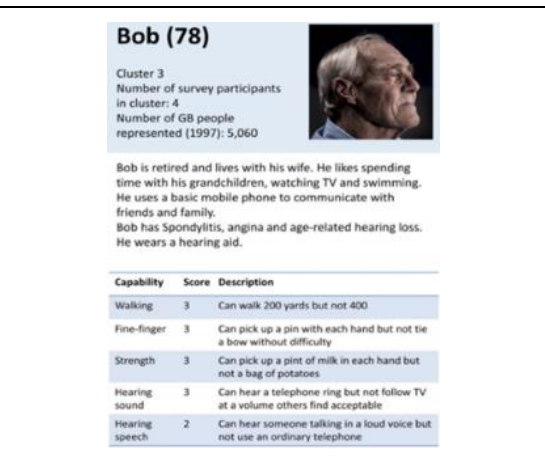 } & 14 & Activises & 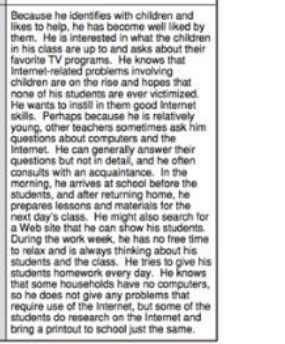 \\
\hline $\begin{array}{l}\text { (a) Persona profile for middle- } \\
\text { aged male and potential customer } \\
\text { of news website [53]. }\end{array}$ & $\begin{array}{l}\text { (b) Persona pr } \\
\text { faced by th } \\
\text { custo }\end{array}$ & $\begin{array}{l}\text { file for medical conditions } \\
\text { potential male elderly } \\
\text { her of a café [16]. }\end{array}$ & $\begin{array}{l}\text { (c) Person } \\
\text { potential custo }\end{array}$ & $\begin{array}{l}\text { a pro } \\
\text { omer }\end{array}$ & $\begin{array}{l}\text { file for teacher and } \\
\text { of educational website } \\
{[18] \text {. }}\end{array}$ \\
\hline
\end{tabular}

Figure 4: Representative examples of personas to inform market research

\subsection{Market research personas}

Figure 4 outlines two different examples of how persona profiles of varying complexity are used for the market case. The authors were interested in increasing users for a news aggregator website [53]. Figure 4a is an example of a simple persona profile for marketing professionals. In this case, the authors were interested in increasing users for a news aggregator website [53]. The 
persona profile contains basic demographic details such as gender and age, and relevant lifestyle factors such as news topic interests and activity on competitors' sites. Meanwhile, Figure $4 \mathrm{~b}$ is an example of a more complex persona profile that can be used for market research purposes. The authors were interested in the factors that would affect visits to a café, as elderly immobility may restrict them from necessary activities such as carrying a tray of food [16]. In addition to quantitative capability data such as scores for vision, hearing, and dexterity, the authors also enriched the persona with a succinct narrative of customers' social life and current assistive equipment usage. The authors report that they recognized the importance of including "the likely situations" of personas with different capability scores [16]. The profiles also contain information on the market size represented by the persona (via "number of GB people represented"). Finally, Figure $4 c$ is an example of a market research persona that is enriched with narrative-like, contextual scenario descriptions [18]. The persona conveys the customer's environment and lifestyle through a detailed narrative that highlights potential opportunities for product usage in different typical contexts.

Such examples give the market research professionals insight into opportunities for introducing and marketing a product to potential customers. As shown in Figure 4, personas developed for market research were primarily focused on general insights on demographics, personality, and lifestyle of potential customers. This focus may be because market segmentation has the goal of producing maximum appeal to target users that "often relies on demographic information to classify" potential customers [44:830]. Two of the eight studies in this category $[28,50]$ also considered income in the persona profile.

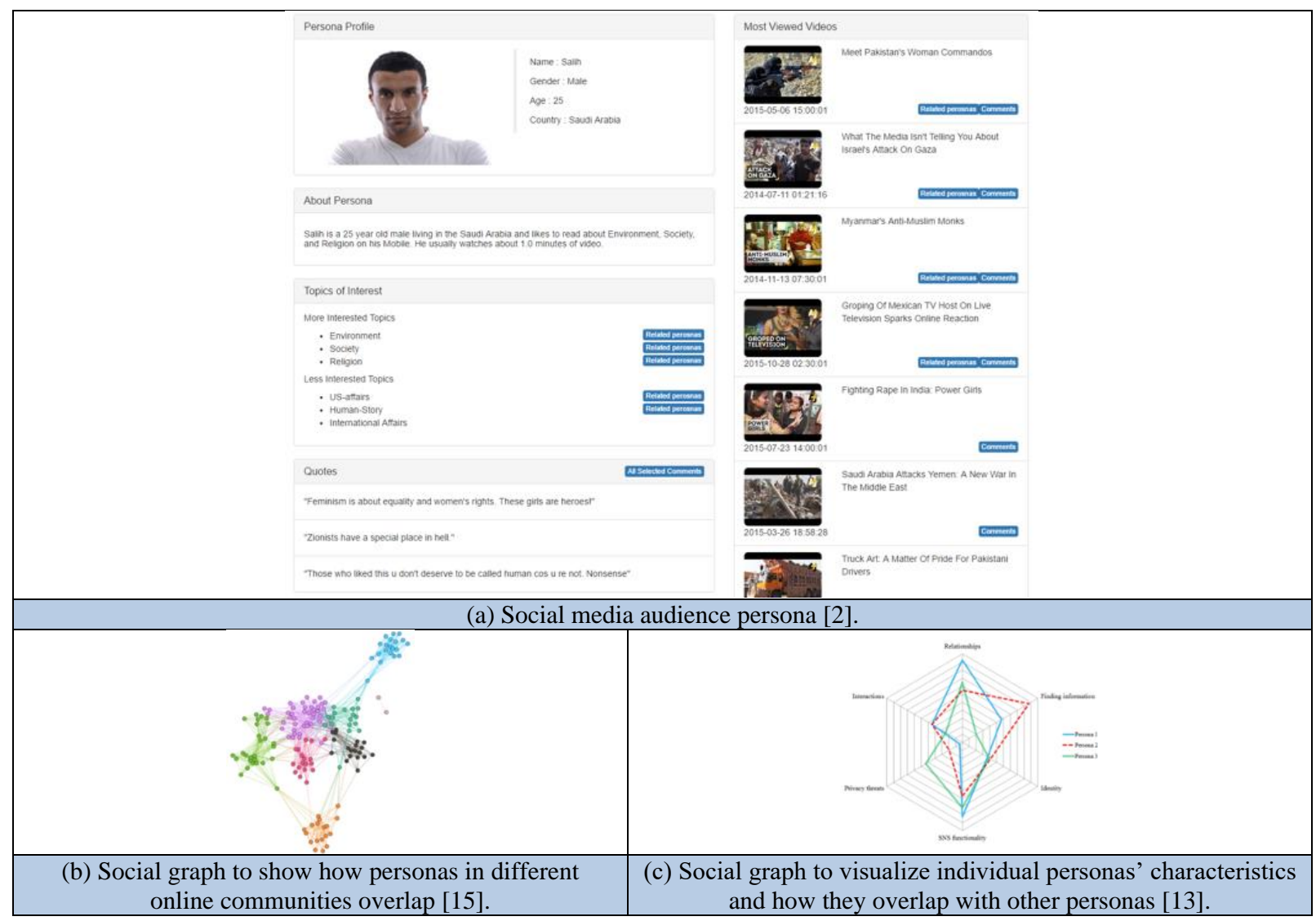

Figure 5: Examples of personas using social media data

\subsection{Social media strategy personas}

For social media strategy personas, four of the six studies were conducted by the same team and used the same analytics data from a YouTube channel [1, 2, 3,
40]. The authors analyzed interactions of users on a popular YouTube channel to develop personas that predict common demographic and viewing activity (duration and content) characteristics. As seen in Figure $5 \mathrm{a}$, the persona profile contains video topics of interest and most commonly viewed videos, in addition to 
information on the device used to visit the YouTube channel [2].

In contrast, the authors that created the graphic in Figure $5 \mathrm{~b}$ were primarily concerned with visualizing overlaps between users of different online communities (Amazon, DBLP, Friendster, LiveJournal, and Orkut) [15]. By "splitting nodes in their "personas," they hoped to "disentangle (..) highly overlapping community structures" of online networks [15:153]. Instead of a traditional persona profile that represents an imaginary archetype, the authors created a "persona graph" with colors representing different online community clusters and lines to represent corresponding overlaps.

Similarly, the authors that created the social graph in Figure 5c wanted to visualize how relationships, activity ("SNS [social network site] functionalities," "interactions," and "finding information"), level of privacy threat, and identity presentation on social media varied and overlapped between different users. Online communities intersected significantly across platforms.

\subsection{Overlaps and differences among personas by professional domain}

Table 1 shows the information that persona profiles from the top four professional domains contain. We quantitatively compared the informational content of the personas by tallying the total pieces of information within each persona layout, reviewing their purposes, and ultimately classifying them under 11 overarching categories (i.e., unique elements): demographics, personality, activity, interests, challenges, environment, hobbies, physical health, mental health, behavioral health, and income. As seen in Table 1, health personas were the only persona category to contain all the unique elements and, as such, the highest number of unique elements among the four domains. UX design and market research personas both contain information from 8 out of 11 of the categories, but the elements presented are not identical. UX design personas include information on user challenges, while market research personas do not; similarly, we found some personas for market research contained information regarding physical health, a category not present in any of the design personas. Finally, social media strategy personas only contain information from four element categories: demographics, personality, activity, and interests.

Furthermore, we can observe that four common elements were present in personas in all four professional domains: demographics, personality, activity, and interests (Figure 6 and Table 2). Also, information on the environment, hobbies, and income could be found among personas in the UX design, health, and market research domains, while information regarding behavioral health was only present in health personas.

Nonetheless, having overlapping elements present among the domains does not necessarily translate to similarities. Rather, the unique elements should be considered in the context of the goals of the specific domains. For example, despite market research and UX design personas sharing many similar elements, market research can be differentiated from UX design due to its greater interest in general market trends and opportunities to recruit new customers [43].

Table 1: Persona information sets (with definitions)

\begin{tabular}{|c|c|c|c|}
\hline \multicolumn{2}{|r|}{ List names } & Number of total elements & Number of unique elements \\
\hline \multicolumn{2}{|r|}{ UX Design } & 60 & $8(\mathrm{~A}, \mathrm{~B}, \mathrm{C}, \mathrm{D}, \mathrm{E}, \mathrm{F}, \mathrm{G}, \mathrm{K})$ \\
\hline \multicolumn{2}{|r|}{ Healthcare } & 35 & $11(\mathrm{~A}, \mathrm{~B}, \mathrm{C}, \mathrm{D}, \mathrm{E}, \mathrm{F}, \mathrm{G}, \mathrm{H}, \mathrm{I}, \mathrm{J}, \mathrm{K})$ \\
\hline \multicolumn{2}{|c|}{ Market Research } & 23 & $8(\mathrm{~A}, \mathrm{~B}, \mathrm{C}, \mathrm{D}, \mathrm{F}, \mathrm{G}, \mathrm{H}, \mathrm{K})$ \\
\hline \multicolumn{2}{|c|}{ Social Media Strategy } & 16 & $4(\mathrm{~A}, \mathrm{~B}, \mathrm{C}, \mathrm{D})$ \\
\hline \multicolumn{2}{|c|}{ Total number of unique element categories } & \multicolumn{2}{|c|}{11 (see below) } \\
\hline & Element & \multicolumn{2}{|c|}{ Description } \\
\hline \multirow{11}{*}{$\begin{array}{l}\text { How the } 11 \\
\text { unique } \\
\text { elements were } \\
\text { categorized: }\end{array}$} & $\mathrm{A}=$ Demographics & \multicolumn{2}{|c|}{ Name, gender, age } \\
\hline & $\mathrm{B}=$ Personality & \multicolumn{2}{|c|}{ Character traits and disposition of the persona } \\
\hline & $\mathrm{C}=$ Activity & \multicolumn{2}{|c|}{ How the persona interacts with the product/ service } \\
\hline & $\mathrm{D}=$ Interests & \multicolumn{2}{|c|}{ Needs and preferences related to product/ service } \\
\hline & $\mathrm{E}=$ Challenges & \multicolumn{2}{|c|}{$\begin{array}{l}\text { Issues preventing persona from full use of the product/ service, i.e., positive } \\
\text { user experience }\end{array}$} \\
\hline & $\mathrm{F}=$ Environment & \multicolumn{2}{|c|}{$\begin{array}{c}\text { Daily context of the persona (e.g., lives in a humid, urban city with public } \\
\text { transport) }\end{array}$} \\
\hline & $\mathrm{G}=$ Hobbies & \multicolumn{2}{|c|}{$\begin{array}{c}\text { Relevant recreational and lifestyle activities, not already represented by }(\mathrm{F}) \\
\text { environment }\end{array}$} \\
\hline & $\mathrm{H}=$ Physical health & \multicolumn{2}{|c|}{ Health condition related to fitness and physical activity } \\
\hline & $\mathrm{I}=$ Mental health & \multicolumn{2}{|c|}{ Health condition related to psychological wellbeing } \\
\hline & $\mathrm{J}=$ Behavioural health & \multicolumn{2}{|c|}{$\begin{array}{l}\text { Health condition related to habits (both physical and mental) that cannot be } \\
\text { fully captured via }(\mathrm{H}) \text { physical health or (I) mental health }\end{array}$} \\
\hline & $\mathrm{K}=$ Income & \multicolumn{2}{|c|}{ Financial circumstances of persona } \\
\hline
\end{tabular}




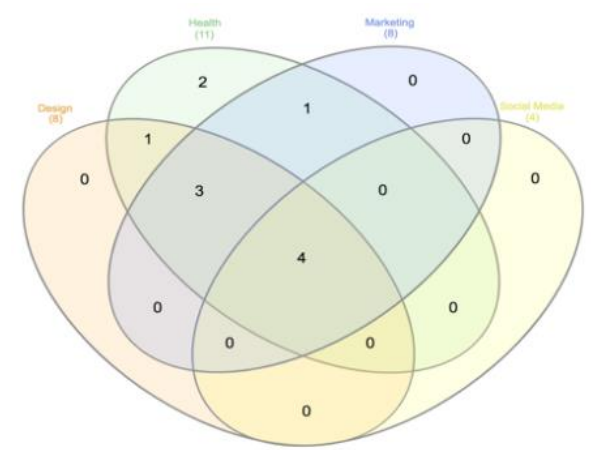

Figure 6: Number of overlapping information types in application domains

Table 2: Overlapping information types in application domains

\begin{tabular}{|c|c|c|}
\hline Professional domains & Total & Elements \\
\hline $\begin{array}{c}\text { UX Design, Healthcare, Market } \\
\text { Research, and Social Media Strategy }\end{array}$ & 4 & $\mathrm{~A}, \mathrm{~B}, \mathrm{C}, \mathrm{D}$ \\
\hline $\begin{array}{c}\text { UX Design, Healthcare, and Market } \\
\text { Research }\end{array}$ & 3 & $\mathrm{~F}, \mathrm{G}, \mathrm{K}$ \\
\hline UX Design, Healthcare & 1 & $\mathrm{E}$ \\
\hline UX Health, Market Research & 1 & $\mathrm{H}$ \\
\hline Healthcare & 2 & $\mathrm{I}, \mathrm{J}$ \\
\hline
\end{tabular}

Conversely, studies related to UX design primarily developed personas to enhance understanding of current users and improve their current experiences to retain their loyalty. As noted by Pruitt \& Grudin [36:11], "marketing and product development have different needs that require different persona attributes, and sometimes different target audiences. Marketing is generally interested in buyer behavior and customers; product development is interested in end users."

Therefore, while all the personas in the studies related to product design emphasized current user activities and goals towards the services in question, such information was only present in a few of the market research studies $[60,65]$ and was nonetheless included for different application purposes. While market research and UX design professionals are, to an extent, concerned with understanding potential and/or current customers to optimize revenues, healthcare professionals are concerned with understanding their potential and/or current patients to enable eradication or long-term management of medical symptoms. Medical researchers are required to cater to their patients their provision of patient-centered services fully, with no profit-driven motives.

Finally, in contrast to the three other domains, the social media strategy domain was distinguished primarily by its data source rather than the distinct principles or goals of the profession. In other words, the personas in this category were shaped by the diverse goals of its creators, and the use of social media data was the only common denominator across the studies.
Indeed, the rise of the Internet has led to low professional barriers of entry for digital content creation [7]. Individuals are using social media for everything from "self-expression, sociability, community engagement, creativity" [27:4] and simultaneously monetizing aspects of their personal lives, blurring the formal line between the personal and the professional. Above all, there is a focus on creating digital content and "influence" via a "personal brand community" with its own culture and codes of behavior [11:344]. Social media strategy personas can, therefore, be regarded as powerful tools for professionals and even "nonprofessionals" from a multitude of disciplines and industries to leverage, with endless applications to the larger populations they seek to engage with.

\section{Discussion}

There is a dire need for systematic processes for persona creation to ensure replicability and maturity in different domains. We found that while there are currently some overlaps in the informational content of personas across different professional domains, such similarities were shallow and distinguished by their applications to each professional domain's particular goals. For example, while UX design, market research, and healthcare all had personas containing content on demographics and lifestyle, this information had distinct purposes and takeaways for the professionals applying them. For design professionals, information on a persona's hobbies informs of potential situations a product and/or service should be designed to accommodate. For marketing professionals, understanding a persona's lifestyle helps them further hone the behavioral activities of a potential market segment, and how best to target them to adopt their services [38]. Finally, for healthcare professionals, considering a persona's social and physical activities are useful insofar as they affect medical conditions [52].

As such, persona profiles must ultimately be systematically developed with the goals of each profession in mind. Based on the studies we evaluated, we can suggest the following general guidelines for professionals seeking to apply personas in specific domains. However, more research is needed to systematically test their applicability in meeting goals.

For professionals interested in understanding the goals and motivations of current and/or end-users, the data collection and subsequent creation of personas should primarily concentrate on the current usage activity of the product category. This specifically refers to how a product or service can provide a usage that is natural for daily life. They may also reflect user 
frustrations, to elucidate current user issues such as "the feeling of inability" (i.e., the user feels they are incapable of using a product) in order to "minimize necessary user operations" [42:1337].

Next, for professionals interested in recruiting new customers and/or understanding general market trends, the data collection and subsequent creation of personas should primarily concentrate on lifestyle, demographic, and, if relevant, geographic information. Such details should anticipate interest and demand for a product among customers [38]. Furthermore, including details on income, or anything concerning customer purchasing power, can help determine customers' ability to access products and/or services [22]. Finally, personas may also include familiarity with the product and/or service, such as work experience in a relevant industry, or loyalty to competitors of the company. This helps to hone in the on interest and demand for a product among customers [38].

For healthcare professionals interested in the physical as well as psychological and lifestyle factors in their assessment of the burden of illnesses, the data collection and subsequent creation of patient personas in for patient care should firstly concentrate on the patient's physical, psychological, and neurocognitive symptoms, in order to determine the clinical evidence of a disease and/or health status [23]. Relatedly, details on lifestyle, such as physical activity and quality of life, can establish the risk of diseases. For example, sexually transmitted infections increase as a result of lifestyle factors such as risky sexual behaviors [23]. If relevant, the patient's familiarity with the disease can also establish the appropriate medical advising language style [51]. Next, the social and living environment of the patient, such as whether they have caretakers at home or need for assistive equipment, can inform the decisions related to patients' rehabilitation outcomes [29]. Finally, the patient's income level can specify patients' ability to access medical services or regimens, such as purchasing certain prescriptions [45].

Finally, our analysis has shown how social media as a tool can be leveraged in endless avenues for professionals and everyday individuals interested in understanding online communities. When exploring social media and big data analytics to build personas, one should consider their specific goals, and how to scale the data, in order to determine what information is relevant and how to effectively extract it [11]. Relatedly, they should also consider how to present relevant information succinctly in the persona by first establishing what information is relevant and how to effectively extract it [11]. If relevant, comparisons and observations of overlaps in users between different online communities can be conducted (as exemplified by Epasto et al. [15]), to gain greater understanding of the online environment social media users interact in and detect unforeseen connections between different communities that they can leverage. This significantly enhances the representativeness of the personas.

\section{Conclusion}

To our knowledge, this is the first review to evaluate the processes of persona creation in different domains and how they pertain to professional goals. We reviewed persona profiles in 51 studies conducted for professional purposes. We identified four of the most common professional domains applying persona creation: UX design, healthcare, market research, and social media strategy. While all the domains we evaluated are interested in understanding their users to enhance service provision, they differ by their specific principles and goals - differences that also distinguish a persona profile's relevance to one profession versus another. UX designers can use personas to enhance endusers' satisfaction with a product. Market researchers can develop personas to further understand and ultimately recruit new customers. Health professionals can develop personas to enhance patient understanding, improve patient care, and empower patients. Digital content creators can build social media strategy personas to more effectively build their online personal brand communities and understand its members. Professionals must, therefore, carefully consider their aims when collecting data and while developing personas. These differing goals naturally result in differences in content among personas, which we evaluated and summarized in our specific recommendations for ideal persona content for each of the four professional domains.

Nonetheless, further research is needed to test how successful personas are in meeting the goals of relevant professionals, as well as to develop systematic methodologies for persona creation in different domains. For example, healthcare persona profiles are only successful if they can aid healthcare professionals in improving patient outcomes, and the success rate for persona creation processes are replicable in different patient cohorts.

\section{References}

[1] An, J., H. Kwak, and B.J. Jansen, "Personas for Content Creators via Decomposed Aggregate Audience Statistics", Proceedings of Advances in Social Network Analysis and Mining (ASONAM 2017), (2017).

[2] An, J., H. Kwak, S. Jung, J. Salminen, and B.J. Jansen, "Customer segmentation using online platforms: isolating behavioral and demographic segments for persona creation 
via aggregated user data", Social Network Analysis and Mining 8(1), 2018.

[3] An, J., H. Kwak, J. Salminen, S. Jung, and B.J. Jansen, "Imaginary People Representing Real Numbers: Generating Personas from Online Social Media Data", ACM Transactions on the Web (TWEB) 12(4), 2018, pp. Article No. 27.

[4] Aoyama, M., "Persona-and-scenario based requirements engineering for software embedded in digital consumer products", Proceedings of the 13th IEEE International Conference on Requirements Engineering (RE'05), (2005), 85-94.

[5] Aoyama, M., "Persona-Scenario-Goal Methodology for User-Centered Requirements Engineering", Proceedings of the 15th IEEE International Requirements Engineering Conference (RE 2007), (2007), 185-194.

[6] Bamman, D., B. O'Connor, and N.A. Smith, "Learning Latent Personas of Film Characters", Proceedings of the 51st Annual Meeting of the Association for Computational Linguistics, (2013), 10.

[7] Blank, G., "Who creates content? Stratification and content creation on the Internet", Information,

Communication \& Society 16(4), 2013, pp. 590-612. [8] Blomquist, A., and M. Arvola, "Personas in action: ethnography in an interaction design team", Proceedings of the second Nordic conference on Human-computer interaction, ACM (2002), 197-200.

[9] Brickey, J., S. Walczak, and T. Burgess, "A Comparative Analysis of Persona Clustering Methods", AMCIS 2010 Proceedings, (2010).

[10] Brooks, C., and J. Greer, "Explaining Predictive Models to Learning Specialists Using Personas", Proceedings of the

Fourth International Conference on Learning Analytics And Knowledge, ACM (2014), 26-30.

[11] Chen, C.-P., "Exploring personal branding on YouTube", Journal of internet commerce 12(4), 2013, pp. 332-347.

[12] Chu, E., P. Vijayaraghavan, and D. Roy, "Learning Personas from Dialogue with Attentive Memory Networks", Proceedings of the 2018 Conference on Empirical Methods in Natural Language Processing, Association for Computational Linguistics (2018), 2638-2646.

[13] Dang-Pham, D., S. Pittayachawan, and M. Nkhoma, "Demystifying online personas of Vietnamese young adults on Facebook: A Q-methodology approach", Australasian Journal of Information Systems 19(0), 2015.

[14] Du, L., and Z. Wang, "Research on the Annual Reading Report of Academic Libraries Based on Personas", Open Journal of Social Sciences 6(10), 2018, pp. 95-105. [15] Epasto, A., S. Lattanzi, and R. Paes Leme, "EgoSplitting Framework: From Non-Overlapping to Overlapping Clusters", Proceedings of the 23rd ACM SIGKDD

International Conference on Knowledge Discovery and Data Mining, ACM (2017), 145-154.

[16] Goodman-Deane, J., S. Waller, D. Demin, A. Gonzálezde-Heredia, M. Bradley, and J.P. Clarkson, "Evaluating Inclusivity using Quantitative Personas", (2018).

[17] Greenhalgh, T., and R. Peacock, "Effectiveness and efficiency of search methods in systematic reviews of complex evidence: audit of primary sources", $B M J$ 331(7524), 2005, pp. 1064-1065.
[18] Hisanabe, Y., "Persona marketing for Fujitsu kids site", Fujitsu Scientific and Technical Journal 45(2), 2009, pp. 210-218.

[19] Holden, R.J., A. Kulanthaivel, S. Purkayastha, K.M. Goggins, and S. Kripalani, "Know thy eHealth user: Development of biopsychosocial personas from a study of older adults with heart failure", International Journal of Medical Informatics 108, 2017, pp. 158-167.

[20] Holmgard, C., A. Liapis, J. Togelius, and G.N. Yannakakis, "Evolving personas for player decision modeling", Computational Intelligence and Games (CIG), 2014 IEEE Conference on, IEEE (2014), 1-8.

[21] Ishii, R., S. Ito, M. Ishihara, T. Harada, and R. Thawonmas, "Monte-Carlo Tree Search Implementation of Fighting Game AIs Having Personas", 2018 IEEE Conference on Computational Intelligence and Games (CIG), IEEE (2018), 1-8.

[22] Jenkinson, A., "Beyond segmentation", Journal of targeting, measurement and analysis for marketing 3(1), 1994, pp. 60-72.

[23] Justice, A.C., C.H. Chang, L. Rabeneck, and R. Zackin, "Clinical importance of provider-reported HIV symptoms compared with patient-report", Medical care, 2001, pp. 397408.

[24] Kanno, T., T. Ooyabu, and K. Furuta, "Integrating Human Modeling and Simulation with the Persona Method", Universal Access in Human-Computer Interaction. Users Diversity, Springer Berlin Heidelberg (2011), 51-60. [25] Kwak, H., J. An, and B.J. Jansen, "Automatic Generation of Personas Using YouTube Social Media Data", Proceedins of the Hawaii International Conference on System Sciences (HICSS-50), (2017), 833-842.

[26] Li, J., M. Galley, C. Brockett, G. Spithourakis, J. Gao, and B. Dolan, "A Persona-Based Neural Conversation Model", Proceedings of the 54th Annual Meeting of the Association for Computational Linguistics (Volume 1: Long Papers), Association for Computational Linguistics (2016), 994-1003.

[27] Livingstone, S., "Taking risky opportunities in youthful content creation: teenagers' use of social networking sites for intimacy, privacy and self-expression", New media \& society 10(3), 2008, pp. 393-411.

[28] Masiero, A.A., M.G. Leite, L.V.L. Filgueiras, and P.T. Aquino Jr., "Multidirectional Knowledge Extraction Process for Creating Behavioral Personas", Proceedings of the 10th Brazilian Symposium on Human Factors in Computing Systems and the 5th Latin American Conference on HumanComputer Interaction, Brazilian Computer Society (2011), 91-99.

[29] McMahon, M., P. Miller, R. Wikoff, M.J. Garrett, and $\mathrm{K}$. Ringel, "Life situations, health beliefs, and medical regimen adherence of patients with myocardial infarction.", Heart \& lung: the journal of critical care 15(1), 1986, pp. $82-86$.

[30] Miaskiewicz, T., T. Sumner, and K.A. Kozar, "A latent semantic analysis methodology for the identification and creation of personas", Proceedings of the SIGCHI

Conference on Human Factors in Computing Systems, ACM (2008), 1501-1510.

[31] Mijač, T., M. Jadrić, and M. Ćukušić, "The potential and issues in data-driven development of web personas", 2018 
41 st International Convention on Information and Communication Technology, Electronics and Microelectronics (MIPRO), (2018), 1237-1242. [32] N. Tu, Q. He, T. Zhang, et al., "Combine Qualitative and Quantitative Methods to Create Persona", 2010 3rd International Conference on Information Management, Innovation Management and Industrial Engineering, (2010), 597-603.

[33] Nielsen, L., K.S. Hansen, J. Stage, and J. Billestrup, “A Template for Design Personas: Analysis of 47 Persona Descriptions from Danish Industries and Organizations", International Journal of Sociotechnology and Knowledge Development 7(1), 2015, pp. 45-61.

[34] Parise, S., and P.J. Guinan, "Marketing using web 2.0", Proceedings of the 41st Annual Hawaii International Conference on System Sciences (HICSS 2008), IEEE (2008), 281-281.

[35] Pruitt, J., and T. Adlin, The Persona Lifecycle: Keeping People in Mind Throughout Product Design, Morgan Kaufmann, Boston, 2006.

[36] Pruitt, J., and J. Grudin, "Personas: Practice and Theory", Proceedings of the 2003 Conference on Designing for User Experiences, ACM (2003), 1-15.

[37] Rahimi, M., and J. Cleland-Huang, "Personas in the Middle: Automated Support for Creating Personas As Focal Points in Feature Gathering Forums", Proceedings of the 29th ACM/IEEE International Conference on Automated Software Engineering, ACM (2014), 479-484.

[38] Revella, Adele, Buyer Personas: How to Gain Insight into Your Customer's Expectations, Align Your Marketing Strategies, and Win More Business, Wiley, 2015.

[39] Salminen, J., K. Guan, S. Jung, S.A. Chowdhury, and B.J. Jansen, "A Literature Review of Quantitative Persona Creation", CHI '20: Proceedings of the 2020 CHI Conference on Human Factors in Computing Systems, ACM (2020), 1-14.

[40] Salminen, J., S. Şengün, H. Kwak, et al., "From 2,772 segments to five personas: Summarizing a diverse online audience by generating culturally adapted personas", First Monday 23(6), 2018.

[41] dos Santos, T.F., D.G. de Castro, A.A. Masiero, and P.T.A. Junior, "Behavioral persona for human-robot interaction: a study based on pet robot", International Conference on Human-Computer Interaction, Springer (2014), 687-696.

[42] von Saucken, C., F. Lachner, and U. Lindemann, "Principles for user experience: what we can learn from bad examples", KEER2014. Proceedings of the 5th Kanesi
Engineering and Emotion Research; International Conference; Linköping; Sweden; June 11-13, Linköping University Electronic Press (2014), 1331-1341.

[43] Scott, D.M., The New Rules of Marketing, John Wiley and Sons, Hoboken, New Jersey, 2007.

[44] Sinha, R., "Persona development for information-rich domains", CHI '03 Extended Abstracts on Human Factors in Computing Systems, ACM (2003), 830-831.

[45] Smeets, R.G., A.M. Elissen, M.E. Kroese, N. Hameleers, and D. Ruwaard, "Identifying subgroups of highneed, high-cost, chronically ill patients in primary care: A latent class analysis", PloS one 15(1), 2020, pp. e0228103. [46] Tanenbaum, M.L., R.N. Adams, E. Iturralde, et al., "From Wary Wearers to d-Embracers: Personas of Readiness to Use Diabetes Devices", Journal of Diabetes Science and Technology 12(6), 2018, pp. 1101-1107.

[47] Tempelman-Kluit, N., and A. Pearce, "Invoking the User from Data to Design", College \& Research Libraries 75(5), 2014, pp. 616-640.

[48] Thoma, V., and B. Williams, "Developing and Validating Personas in e-Commerce: A Heuristic Approach", Human-Computer Interaction - INTERACT 2009, Springer Berlin Heidelberg (2009), 524-527.

[49] Torgerson, C., Systematic Reviews, A\&C Black, 2003.

[50] Tu, N., X. Dong, P.P. Rau, and T. Zhang, "Using cluster analysis in Persona development", 2010 8th International Conference on Supply Chain Management and Information, (2010), 1-5.

[51] Vosbergen, S., J.M.R. Mulder-Wiggers, J.P. Lacroix, et al., "Using personas to tailor educational messages to the preferences of coronary heart disease patients", Journal of Biomedical Informatics 53, 2015, pp. 100-112.

[52] Wang, L., L. Li, H. Cai, L. Xu, B. Xu, and L. Jiang, "Analysis of Regional Group Health Persona Based on Image Recognition”, 2018 Sixth International Conference on Enterprise Systems (ES), (2018), 166-171.

[53] Watanabe, Y., H. Washizaki, K. Honda, et al., "ID3P: Iterative Data-driven Development of Persona Based on Quantitative Evaluation and Revision", Proceedings of the 10th International Workshop on Cooperative and Human Aspects of Software Engineering, IEEE Press (2017), 49-55. [54] Wöckl, B., U. Yildizoglu, I. Buber, B. Aparicio Diaz, E. Kruijff, and M. Tscheligi, "Basic Senior Personas: A Representative Design Tool Covering the Spectrum of European Older Adults", Proceedings of the 14th International ACM SIGACCESS Conference on Computers and Accessibility, ACM (2012), 25-32. 